\title{
Variabilité des descripteurs pluviométriques intrasaisonniers à impact agricole dans le bassin cotonnier de Côte d'Ivoire : cas des zones de Boundiali, Korhogo et Ouangolodougou
}

\author{
Charles Sékpa DEKOULA 1,2*, Brou KOUAME 1, Kouadio Emmanuel N'GORAN 1, Jean-Noël \\ EHOUNOU 1,2, Guy Fernand YAO 1, Koffi Emmanuel KASSIN 1, Julien Brou KOUAKOU 1, Angelo \\ Evariste Badou N'GUESSAN ${ }^{1}$, et Nagnin SORO ${ }^{2}$ \\ ${ }_{1}^{1}$ Centre National de Recherche Agronomique (CNRA), Laboratoire Central Sols, Eaux et Plantes, Programme \\ Gestion Durable des Sols et Maîtrise de l'Eau, Programme coton, 01 BP 633 Bouaké 01, Côte d'Ivoire \\ 2 Université Félix Houphouët-Boigny de Cocody-Abidjan, Unité de Formation et de Recherche (UFR) des Sciences \\ de la Terre et des Ressources Minières (STRM), Laboratoire de Sciences et Techniques de l'Eau et de \\ l'Environnement (LSTEE), 22 BP 582 Abidjan 22, Côte d'Ivoire \\ ${ }^{*}$ Auteur correspondant, E-mail : dekoula@gmail.com ; Tel : (+225) 09485856 / (+225) 79101373
}

Original submitted in on $8^{\text {th }}$ August 2018. Published online at www.m.elewa.org on $31^{\text {st }}$ October 2018 https://dx.doi.org/10.4314/jab.v130i1.7

\section{RÉSUME}

Objectif : Cette étude vise à caractériser l'évolution des descripteurs pluviométriques intrasaisonniers à impact agricole durant la saison culturale dans le bassin cotonnier ivoirien. A cet effet,

Méthodologie et résultats: dix descripteurs pluviométriques intrasaisonniers susceptibles d'influencer l'agriculture ont été analysés à partir des données pluviométriques couvrant les périodes 1951-2000 et 2011-2016 et provenant des bases de données de la Société de Développement et d'Exploitation Aéroportuaire, Aéronautique et Météorologique et du Centre National de Recherche Agronomique. Les tests de tendance (Mann-Kendall) et de stationnarité (Pettitt) et une comparaison des périodes ont été appliqués aux variables déterminées. L'analyse des cumuls pluviométriques saisonniers, du nombre de jours de pluie et des précipitations moyennes journalières a mis en évidence une tendance significative à la baisse de ces paramètres sur la période 1951-2000. Les fins et les longueurs de la saison des pluies ont présenté respectivement une tendance significative à la précocité et au raccourcissement. Les autres descripteurs intrasaisonniers ont peu varié. En revanche, des ruptures statistiquement significatives ont été observées entre 1968 et 1979. La comparaison des périodes indique une dégradation des conditions climatiques pendant la période sèche (1971-2000). Ces dégradations se sont accentuées dans la période actuelle (2011-2016).

Conclusion et application: Les nouvelles conditions climatiques observées dans le bassin cotonnier traduites par une variabilité des descripteurs intrasaisonniers rendent de plus en plus difficile la planification agricole. Dès lors, l'actualisation des calendriers culturaux dans le bassin cotonnier devient nécessaire.

Mots-clés : Descripteurs intrasaisonniers, pluviométrie, agriculture, bassin cotonnier, Côte d'Ivoire. 


\section{ABSTRACT}

Objective: This study aims to characterize the evolution of agricultural impact intra-seasonal descriptors during the growing season in cotton production area of Côte d'Ivoire. For this purpose,

Methodology and results: ten intraseasonal descriptors likely to influence agriculture were determined from rainfall data covering the periods 1951-2000 and 2011-2016 from the Airport, Aeronautical and Meteorological Development and Operations Company and the National Agronomic Research Center. Trend (Mann-Kendall) and stationarity (Pettitt) tests and an inter-period comparison were applied to the identified variables. Analysis of seasonal rainfall accumulations, number of rainy days and average daily rainfall amounts showed a significant downward trend over the period 1951-2000. The cessation and length of the rainy season showed a significant trend towards early onset and shortening respectively. Other intraseasonal descriptors do not varied significantly. In contrast, statistically significant breaks were observed between 1968 and 1979. The period comparison indicated a deterioration in conditions during the dry period (1971-2000). The comparison of the periods indicates a deterioration of the climatic conditions during the dry period (1971-2000). These degradations have increased in the current period (2011-2016).

Conclusion and application: New climatic conditions observed in the cotton production area translated by a variability of intraseasonal descriptors are making agricultural planning increasingly difficult. It is therefore necessary to update the crop calendars in the cotton production area.

Keywords: Intraseasonal descriptors, rainfall, agriculture, cotton production area, Côte d'Ivoire.

\section{INTRODUCTION}

Depuis les années 1970, l'Afrique de l'Ouest a subi une des plus fortes variations pluviométriques observées à l'échelle du globe. Cette variabilité climatique a non seulement affecté le régime des précipitations, mais également les ressources hydrologiques et végétales (Aguiar, 2009). L'agriculture qui occupe une place prépondérante dans les économies nationales reste une des activités les plus affectées par ces variations du climat. En effet, dans cette région de l'Afrique, l'agriculture est majoritairement de type pluvial. Par conséquent, les performances des systèmes de productions sont étroitement soumises au climat (Alhassane et al., 2013). De nombreuses études sur la variabilité climatique à l'échelle de la Côte d'Ivoire (Diomandé et al., 2013 ; Balliet et al., 2016 et Dekoula et al., 2018), ont montré des modifications pluviométriques souvent marquées par des baisses relativement brutales des cumuls annuels dans l'ensemble du pays. La plupart de ces études ont plus été menées à l'échelle hydrologique qu'à l'échelle agronomique. Aussi, les cumuls pluviométriques annuels ou mensuels ont-ils été considérés comme des variables représentatives de la qualité de la saison des pluies. En agriculture, réduire la qualité de la saison des pluies à son simple cumul ou à sa durée serait insuffisant pour une bonne compréhension de la saison (Boyard, 2013). En effet, une saison qui présente un fort cumul peut avoir une mauvaise répartition spatio-temporelle des pluies et contenir de longs épisodes secs potentiellement néfastes au bon développement des cultures s'ils interviennent durant la phase de floraison (Sultan et al., 2005). Cette situation rend de plus en plus difficile la planification agricole, surtout celle des semis. De plus, le nouveau contexte climatique induit également la variabilité des descripteurs pluviométriques intrasaisonniers tels que les dates de démarrage, de fin et la longueur de la saison des pluies potentiellement utiles (Noufé et al., 2015). On note également des occurrences d'épisodes secs de plus en plus longs durant la saison des pluies utiles à la production agricole. L'agriculture ivoirienne étant essentiellement pluviale, les modifications des éléments descriptifs de la saison des pluies entrainent des perturbations sur la production des cultures notamment sur le rendement. Selon Ahoussi et al., (2013), les perturbations des systèmes de cultures existants tels que les calendriers culturaux sont d'ores et déjà observées dans plusieurs localités du pays et les exploitants agricoles en sont conscients. Or les activités 
agricoles, qui contribuent à l'approvisionnement en aliments des populations, constituent aussi la première source de leurs revenus. Cette forte dépendance climatique de l'agriculture sera aggravée par l'accentuation de la variabilité pluviométrique dans plusieurs zones agricoles de la Côte d'Ivoire comme celle de la zone de production du coton. Comprendre aujourd'hui la structure de la saison des pluies potentiellement

\section{MATÉRIEL ET MÉTHODES}

Zone d'étude: Le district des savanes fait partie intégrante du bassin cotonnier ivoirien. C'est l'une des zones de fortes productions de coton de la Côte d'Ivoire (Le Guen, 2004). La production moyenne en coton graine de ces dix dernières années de cette zone représente environ deux tiers de la production nationale estimée à 300316 tonnes (Intercoton, 2018). Située entre les longitudes $3^{\circ} 50$ et $7^{\circ}$ ouest et les latitudes $8^{\circ} 50$ et $10^{\circ}$ nord, elle délimite un ensemble géographique constitué de 3 régions, notamment la utiles pour l'agriculture et son évolution, serait une ébauche de solutions aux problèmes rencontrés par les exploitants agricoles et un outil d'aide à la décision pour orienter les actions d'investissement agricole. La présente contribution se propose donc d'améliorer la compréhension sur les variations des descripteurs pluviométriques intrasaisonniers à impact agricole dans le bassin cotonnier ivoirien.

Bagoué, le Poro et le Tchologo (figure 1). Deux saisons bien marquées caractérisent le climat de cette zone : la saison sèche en hiver boréal (de novembre à avril) et la saison des pluies en été (de mai à octobre). La pluviométrie moyenne interannuelle est inférieure à $1200 \mathrm{~mm}$ (Brou, 2005). La pédologie de cette zone est caractérisée par trois sous classes de sols ferralitiques; les sols sur roches basiques, les sols ferrugineux tropicaux et les sols hydromorphes (Koné, 2007).

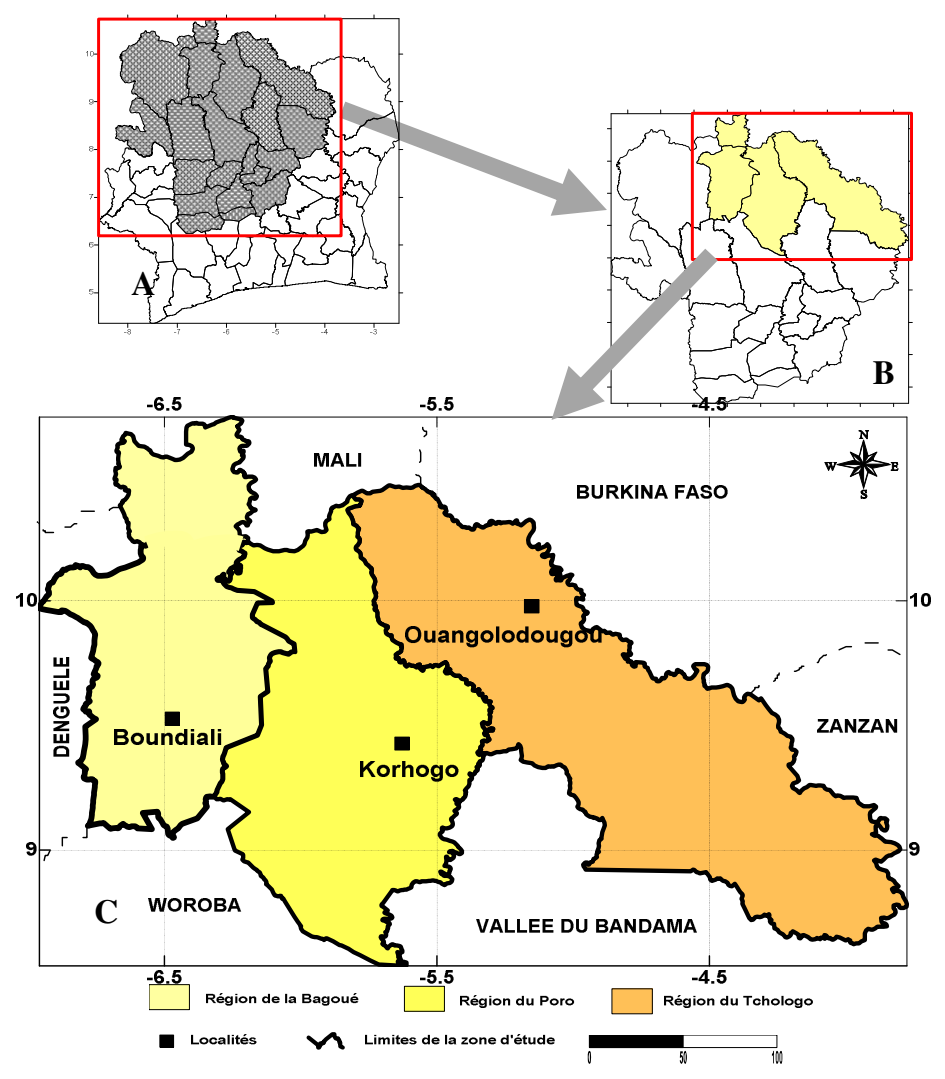

Figure 1 : Localisation de la zone d'étude en Côte d'Ivoire (A : Côte d'Ivoire, B : Bassin cotonnier et C : District des savanes) 
Données pluviométriques: Les analyses pluviométriques se basent sur les précipitations journalières enregistrées dans trois localités (Boundiali, Korhogo et Ouangolodougou) et se concentrent sur deux séries chronologiques: 1951-2000 et 2011-2016. Le choix de ces deux séries est imposé par le manque de données dû à la crise militaro-politique entre 2002 et 2010. Les données de ces séries proviennent de la banque de données de la Société d'Exploitation et de Développement Aéroportuaire, Aéronautique et Météorologique (SODEXAM) et du Laboratoire Central Sols, Eaux et Plantes (LCSEP) du Centre National Recherche Agronomique (CNRA).

Définition des paramètres étudiés : La qualité d'une saison des pluies ne peut être appréhendée à partir de son seul cumul saisonnier ou encore de sa durée. En réalité, une saison des pluies est structurée autour de variables pluviométriques qui construisent le cumul saisonnier (Boyard, 2013). C'est ainsi que des critères ont été définis pour la détermination des variables appelés descripteurs intrasaisonniers (DIS) qui structurent la saison des pluies. Le début de la saison des pluies (DSP) et la fin de la saison des pluies (FSP) ont été calculées à partir des définitions agroclimatiques proposées par Sivakumar (1988) et basées sur des seuils empiriques de précipitations. Cette méthode a été adaptée aux spécificités de la Côte d'Ivoire par Sarr (2007) et Goula et al. (2010). Les critères retenus sont une légère modification apportée à ceux de Goula et al, (2010) par Dekoula et al., (2018). Ainsi pour la zone étudiée :

le DSP a été déterminé selon le critère suivant: la saison des pluies démarre à partir du 15 mars, lorsqu'on enregistre au moins $20 \mathrm{~mm}$ de pluie sur 2 jours consécutifs sans épisodes secs de plus de 7 jours dans les 30 jours qui suivent la date indiquée.

la FSP intervient lorsqu' à partir du 1 er octobre, un sol susceptible de contenir $70 \mathrm{~mm}$ d'eau de pluie disponible aura complètement épuisé ce stock suite à une perte quotidienne de $4 \mathrm{~mm}$ par évapotranspiration. Par ailleurs, cette date obtenue doit être suivie d'épisode sec d'au moins 20 jours.

La longueur de la saison des pluies (LSP) est obtenue par la différence entre la FSP et le DSP, soit :

$$
L S P-F S P-D S P
$$

Le cumul pluviométrique saisonnier (CPS) est déterminé par la quantité de pluies recueillie au cours de la saison. Moron et al. (2007) suggèrent qu'un cumul pluviométrique saisonnier puisse se décomposer comme le produit du nombre de jours de pluie supérieur à $1 \mathrm{~mm}$ durant la saison des pluies (NJP) et de la hauteur journalière moyenne des précipitations (HMP) :

$C P S=N J P \times H M F$

Les séquences sèches (SS) des mois de juin, juillet, août et septembre ont été déterminées car c'est au cours de ces mois que se développent les phases phénologiques (tallage, floraison, formation et remplissage des capsules) les plus critiques des plantes. Une séquence sèche est en effet, définie comme étant le nombre de jours consécutifs sans pluie de hauteur supérieure à la valeur minimale $(1 \mathrm{~mm})$ de la plus petite des classes des hauteurs journalières de précipitations proposée par les normes internationales définies par l'Organisation Météorologique Mondiale (OMM, 1990). Les différentes classes sont définies en fonction du nombre de jours de pluie de hauteur comprise entre : 1 et $10 \mathrm{~mm}$ (P1) ; 10 et $30 \mathrm{~mm}$ (P2) ; 30 et $50 \mathrm{~mm}$ (P3) ; >50 mm (P4). Un ensemble de descripteurs intrasaisonniers susceptible d'influencer les activités agricoles a été retenu pour les différentes analyses (tableau 1). Le logiciel Instat + V.3.037 (Stern et al., 2006) a servi au calcul des DIS et les analyses statistiques.

Analyse des tendances: L'analyse des tendances des descripteurs intrasaisonniers a été réalisée pour chaque station de 1951 à 2000. Le test de MannKendall a été appliqué afin de détecter la présence d'une tendance monotone au sein des séries chronologiques en l'absence de toute saisonnalité. II s'agit d'un test non paramétrique dérivant des travaux de Mann (1945) et Kendall (1975). Ce test est basé sur l'hypothèse nulle $\mathrm{H}_{0}$ qu'll n'y a pas de tendance dans la série. L'hypothèse alternative $\left(H_{1}\right)$, désigne la nonstationnarité de la série. Elle est complémentaire à $\mathrm{H}_{0}$ et correspond aux hypothèses alternatives d'une évolution négative ou positive. Par ailleurs, il a été nécessaire de calculer la probabilité associée à la taille de l'échantillon afin de quantifier statistiquement l'ampleur de la tendance. La significativité du test est représentée par la probabilité de détecter une tendance dans la série avec une erreur inférieure ou égale à $5 \%$. 
Dekoula et al., J. Appl. Biosci. 2018 Variabilité des descripteurs pluviométriques intrasaisonniers à impact agricole dans le bassin cotonnier de Côte d'Ivoire : zones de Boundiali, Korhogo et Ouangolo

Tableau 1 : Descripteurs pluviométriques intrasaisonniers définis pour analyse

\begin{tabular}{llll}
\hline Acronyme & Nom du descripteur & Définition du descripteur & Unité \\
\hline DSP & Début de la saison des pluies & Démarrage des pluies utiles en agriculture & Date \\
\hline FSP & Fin de la saison des pluies & Fin des pluies utiles en agriculture & Date \\
\hline LSP & Longueur de la saison des pluies & Durée de la saison des pluies & Jours \\
\hline CPS & Cumul pluviométrique saisonnier & $\begin{array}{l}\text { Quantité de précipitations enregistrée entre le } \\
\text { début et la fin de la saison des pluies }\end{array}$ & $\mathrm{mm}$ \\
\hline NJP & Nombre de jours de pluies & $\begin{array}{l}\text { Nombre de jours ayant enregistré une pluie } \\
\text { supérieure à 1 mm }\end{array}$ & Jours \\
\hline HMP & $\begin{array}{l}\text { Hauteur journalière moyenne } \\
\text { des précipitations }\end{array}$ & $\begin{array}{l}\text { Quantité journalière moyenne de précipitations } \\
\text { enregistrées par jour pluvieux }\end{array}$ & $\mathrm{mm} /$ jour \\
\hline SS $_{\mathrm{jn}}$ & Séquence sèche en juin & $\begin{array}{l}\text { Nombre de jours sans pluie le plus élevé entre } \\
\text { deux pluies consécutives en juin }\end{array}$ & Jours \\
\hline SS $_{\mathrm{jt}}$ & Séquence sèche en juillet & $\begin{array}{l}\text { Nombre de jours sans pluie le plus élevé entre } \\
\text { deux pluies consécutives en juillet }\end{array}$ & Jours \\
\hline SS $_{\mathrm{au}}$ & Séquence sèche en août & $\begin{array}{l}\text { Nombre de jours sans pluie le plus élevé entre } \\
\text { deux pluies consécutives en août }\end{array}$ & Jours \\
\hline SS $_{\mathbf{s p}}$ & Séquence sèche en septembre & $\begin{array}{l}\text { Nombre de jours sans pluie le plus élevé entre } \\
\text { deux pluies consécutives en septembre }\end{array}$ & Jours \\
\hline
\end{tabular}

Analyse des ruptures : Il a été possible de déterminer la non-stationnarité de ces séries au cours de la période 1951-2000 et d'isoler les périodes d'anomalies. Le test non-paramétrique de Pettitt (1979), capable d'estimer la position d'un changement de moyenne ou point de rupture dans une série a été appliqué. Les paramètres utilisés pour ce test ont été : l'hypothèse alternative $\neq 0$, la rupture est considérée comme étant significative lorsque la probabilité associée est inférieure à $5 \%(p<0,05)$ le nombre de simulations est 10000 et le temps maximum de simulation est $180 \mathrm{~s}$ (Addinsoft, 2016).

Analyse statistique d'inter-comparaison des descripteurs intrasaisonniers: En vue de faire ressortir les changements et variations dans l'évolution des descripteurs intrasaisonniers à impact agricole, des périodes ont été considérées. La période 1951-2000 a été scindée en deux périodes 1951-1970 et 1971-2000, respectivement en tant que périodes humide et sèche (Kouassi et al., 2010,). L'année 1970 a été retenue car,

\section{RÉSULTATS}

Tendances des descripteurs intrasaisonniers: Le tableau 2 présente les valeurs moyennes et les tendances décennales des descripteurs intrasaisonniers enregistrées sur la période 1951-2000 à Boundiali, Korhogo et Ouangolodougou. Le DSP oscille en moyenne entre le 1er mai et le 22 mai. Les tendances décennales sont au retard de 1 à 4 jours mais restent non significatives. La FSP a lieu en c'est à cette année que la majorité des auteurs s'accorde à reconnaitre que la rupture pluviométrique s'est globalement produite en Côte d'Ivoire (Noufé, 2011). La période 2011-2016 a été retenue comme période actuelle marquant la reprise des opérations de collecte de données dans les différents postes de mesures dans le bassin cotonnier. Pour comparer les périodes de référence le test de Student $(t)$ de comparaison de la moyenne a été utilisé. Ce test paramétrique permet de déterminer la significativité ou non d'une différence entre deux moyennes selon le seuil ( $\alpha$ ) de significativité choisi pour cette étude qui est $\alpha=5 \%$. La différence est dite très significative si la probabilité $p$ du test $t$ appliqué est inférieure à 0,01 et significative si elle est comprise dans l'intervalle $[0,01$ 0,05 . Les différences sont alors dites non significatives, lorsque la probabilité $p$ du test $t$ dépasse le seuil de 0,05 . Le programme avancé XLSTAT-Premium d'Addinsoft du tableur Excel a été utilisé pour les tests et procédures statistiques.

moyenne entre le 30 octobre et le 8 novembre. Le tableau 2 montre une tendance à la précocité de -2 à -3 jours par décennie. Cette tendance n'est statistiquement significative qu'à Korhogo. La LSP est comprise entre 162 jours et 188 jours avec une tendance au raccourcissement dans toutes les localités. Cette tendance (-2 à -6 jours par décennie) n'a été statistiquement significative qu'à 
Ouangolodougou. Les valeurs moyennes des autres DIS ont connu une tendance générale à la baisse, notamment le CPS (variant entre $946 \mathrm{~mm}$ et $1276 \mathrm{~mm}$ ), le NJP (variant entre 51 et 76 jours) et la HMP (variant de 15 à $20 \mathrm{~mm} /$ jour pluvieux). Pour le CPS et le NJP, les tendances à la baisse à Korhogo ($50 \mathrm{~mm}$ par décennie et -4 jours par décennie) et à Ouangolodougou (-102 mm par décennie et -4 jours par décennie) ont été respectivement significatives et très significatives. Quant à la HMP, la tendance à la baisse est statistiquement significative uniquement dans la zone de Boundiali (-1 mm/j par décennie). Les SS des mois de juin, juillet, août et septembre ont varié en moyenne entre 3 et 8 jours dans l'ensemble des localités. Les tendances sont de 1 jour par décennie. En septembre, des tendances à la hausse statistiquement significatives ont été enregistrées à Korhogo et Ouangolodougou. Cependant, pour la station de Boundiali, la tendance statistiquement significative n'a été enregistrée qu'au mois de juillet.

Tableau 2 : Moyennes et tendances décennales des descripteurs intrasaisonniers établies sur la période de 19512000 sur les trois stations

\begin{tabular}{|c|c|c|c|c|c|c|c|}
\hline \multirow[b]{2}{*}{ Indices } & \multirow[b]{2}{*}{ Unité } & \multicolumn{2}{|c|}{ Boundiali } & \multicolumn{2}{|c|}{ Korhogo } & \multicolumn{2}{|c|}{ Ouangolodougou } \\
\hline & & $\begin{array}{c}\text { Moyenne } \\
1951-2000\end{array}$ & $\begin{array}{c}\text { Tendance / } \\
10 \text { ans }\end{array}$ & $\begin{array}{c}\text { Moyenne } \\
1951-2000\end{array}$ & $\begin{array}{c}\text { Tendance / } \\
10 \text { ans }\end{array}$ & $\begin{array}{c}\text { Moyenne } \\
1951-2000\end{array}$ & $\begin{array}{c}\text { Tendance } / \\
10 \text { ans }\end{array}$ \\
\hline DSP & Jours & 15 mai & 1 & 1er mai. & 2 & 22 mai & 4 \\
\hline FSP & Jours & 8 nov. & -2 & 6 nov. & $-3^{*}$ & 30 oct. & -2 \\
\hline LSP & Jours & 175 & -2 & 188 & -3 & 162 & $-6^{*}$ \\
\hline CPS & $\mathrm{mm}$ & 1276 & -72 & 1128 & $-50^{*}$ & 946 & -102 ** \\
\hline NJP & Jours & 64 & -1 & 76 & $-4^{*}$ & 51 & -4 ** \\
\hline HMP & $\mathrm{mm} / \mathrm{jour}$ & 20,3 & $-1^{*}$ & 15 & -1 & 19 & 1 \\
\hline $\mathrm{ES}_{\mathrm{jn}}$ & Jours & 7 & -1 & 6 & -1 & 8 & 1 \\
\hline$E_{j t}$ & Jours & 8 & $-1^{*}$ & 6 & 1 & 8 & 1 \\
\hline $\mathrm{ES}_{\mathrm{au}}$ & Jours & 5 & -1 & 5 & -1 & 6 & 1 \\
\hline $\mathrm{ES}_{\mathrm{sp}}$ & Jours & 5 & 1 & 3 & $1^{*}$ & 5 & $1^{*}$ \\
\hline
\end{tabular}

* tendance significative ; ${ }^{* *}$ tendance très significative $(p<0,05)$.

Point de rupture des descripteurs pluviométriques intrasaisonniers: Les résultats du test de Pettitt (1979) sont résumés dans le tableau 3. Les points de ruptures détectés, présentent des variations beaucoup plus hétérogènes et se situent majoritairement entre 1962 et 1984 . Cependant, les ruptures les plus significatives sont enregistrées entre 1968 et 1979. Le DSP montre une rupture significative à Ouangolodougou (observée en 1968), indiquant un retard après cette date. Au niveau de la LSP, la rupture statistiquement significative a été détectée à Ouangolodougou en 1968. Elle indique un raccourcissement de la LSP après la rupture. Les deux autres localités ne présentent pas de rupture significative. Les changements les plus importants sont observés dans les séries du CPS, du NJP et de l'HMP. Le CPS indique des ruptures significatives à Korhogo en 1973 et présente des ruptures très significatives en 1974 à Boundiali et en 1969 à Ouangolodougou. Au niveau du NJP, 1972 représente l'année de rupture à
Boundiali (significative) et à Korhogo (très significative). À Ouangolodougou, une rupture très significative est indiquée en 1969. Dans les séries de la HMP, Boundiali est la seule localité qui a connu une rupture en 1979 (très significative). Les séquences sèches au cours des différents mois ont des tendances stationnaires dans l'ensemble. Cependant, quand il existe un point de rupture, celui-ci est statistiquement peu significatif.

Comparaisons des périodes 1951-1970,1971-2000 et 2011-2016 : Les pourcentages des variations et leurs niveaux de significativité statistique révélés du test de Student sont présentés dans les tableaux 4,5 et 6 . A Boundiali, les descripteurs FSP, CPS et NJP montrent les variations les plus importantes avec des différences statistiquement significatives entre les périodes: humide versus (vs) sèche et humide vs actuelle. La FSP des périodes sèche (1971-2000) et actuelle (20112016) a été significativement précoce, respectivement de $-2,5 \%$ (8 jours) et $-4,7 \%$ ( 15 jours) par rapport à la période humide (1951-1970). 
Dekoula et al., J. Appl. Biosci. 2018 Variabilité des descripteurs pluviométriques intrasaisonniers à impact agricole dans le bassin cotonnier de Côte d'Ivoire : zones de Boundiali, Korhogo et Ouangolo

Tableau I : Dates des ruptures et des tendances mises en évidence par le test de Pettitt (1979) pour les trois stations

\begin{tabular}{|c|c|c|c|c|c|c|}
\hline & \multicolumn{2}{|r|}{ Boundiali } & \multicolumn{2}{|r|}{ Korhogo } & \multicolumn{2}{|c|}{ Ouangolodougou } \\
\hline Variables & Rupture & $\begin{array}{c}\text { Période de } \\
\text { stationnarité } \\
\text { (Moyenne de la } \\
\text { période) }\end{array}$ & Rupture & $\begin{array}{c}\text { Période de } \\
\text { stationnarité } \\
\text { (moyenne de la } \\
\text { période) }\end{array}$ & Rupture & $\begin{array}{c}\text { Période de } \\
\text { stationnarité } \\
\text { (moyenne de la } \\
\text { période) }\end{array}$ \\
\hline \multirow{2}{*}{ DSP } & \multirow{2}{*}{-} & \multirow[t]{2}{*}{ rocosci } & \multirow[b]{2}{*}{$1973^{4}()$} & 1951-1972 (24 avr.) & \multirow{2}{*}{$1968^{\wedge} *$} & 1951-1967 (8 mai) \\
\hline & & & & 1973-2000 (2 mai) & & 1968-2000 (26 mai) \\
\hline \multirow[b]{2}{*}{ FSP } & \multirow[b]{2}{*}{1974 () } & 1951-1973 (12 nov.) & \multirow[b]{2}{*}{1976 ( ()} & 1951-1975 (10 nov.) & \multirow[b]{2}{*}{ 1977() } & 1951-1976 (4 nov.) \\
\hline & & 1974-2000 (3 nov.) & & 1976-2000 (2 nov.) & & 1977-2000 (27 oct.) \\
\hline \multirow{2}{*}{ LSP } & \multirow[b]{2}{*}{ 1972 () } & 1951-1971 (188 J) & \multirow[b]{2}{*}{ 1973 () } & 1951-1972 (198 J) & \multirow[b]{2}{*}{$1968 *$} & 1951-1967 (181 J) \\
\hline & & $1972-2000(170 \mathrm{~J})$ & & $1973-2000(186 \mathrm{~J})$ & & 1968-2000 (154 J) \\
\hline \multirow{2}{*}{ CPS } & \multirow[b]{2}{*}{$1974 \mathrm{r}^{\star *}$} & $1951-1973(1466 \mathrm{~mm})$ & \multirow[b]{2}{*}{$1973 \nabla^{*}$} & $1951-1972(1225 \mathrm{~mm})$ & \multirow[b]{2}{*}{$1969 \nabla^{* *}$} & $1951-1968(1158 \mathrm{~mm})$ \\
\hline & & $1974-2000(1108 \mathrm{~mm})$ & & $1973-2000(1060 \mathrm{~mm})$ & & $1969-2000(830 \mathrm{~mm})$ \\
\hline \multirow{2}{*}{ NJP } & \multirow[b]{2}{*}{$1972 \gamma^{*}$} & 1951-1971 (71 J) & \multirow[b]{2}{*}{1972 ॠ* } & 1951-1971 (84 J) & \multirow[b]{2}{*}{$1969_{\curlyvee}^{* *}$} & 1951-1968 (58 J) \\
\hline & & $1972-2000(60 \mathrm{~J})$ & & $1972-2000(70 \mathrm{~J})$ & & $1969-2000$ (47 J) \\
\hline \multirow{2}{*}{ HMP } & \multirow{2}{*}{1979 ** } & $1951-1979(22 \mathrm{~mm} / \mathrm{j})$ & \multirow{2}{*}{-} & \multirow[t]{2}{*}{ (1) } & \multirow{2}{*}{-} & \multirow{2}{*}{-} \\
\hline & & $1979-2000(17 \mathrm{~mm} / \mathrm{j})$ & & & & \\
\hline$E S_{j n}$ & - & - & - & - & - & - \\
\hline \multirow{2}{*}{$\mathrm{ES}_{\mathrm{jt}}$} & \multirow[b]{2}{*}{ 1984() } & $1951-1983(9 \mathrm{~J})$ & \multirow{2}{*}{ - } & \multirow{2}{*}{-} & \multirow{2}{*}{ - } & 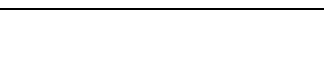 \\
\hline & & $1984-2000(5 \mathrm{~J})$ & & & & - \\
\hline FS & & $1951-1983$ (5,37 J) & & - & 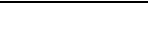 & 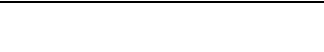 \\
\hline $\mathrm{ES}_{\mathrm{au}}$ & 1984 () & $1984-2000(3,6 \mathrm{~J})$ & & - & - & - \\
\hline FS & & & & $1951-1961(3 \mathrm{~J})$ & & $1951-1979(5 \mathrm{~J})$ \\
\hline$E S_{s p}$ & - & - & $1962^{4}()$ & $1962-2000(4 \mathrm{~J})$ & $1980 \uparrow()$ & $1980-2000$ (7 J) \\
\hline
\end{tabular}

Le CPS des périodes sèche et actuelle a baissé significativement de $-14,4 \%$ et de $-24,4 \%$ par rapport à la période humide. Le NJP de la période sèche a baissé de $-15,5 \%$ par rapport à la période humide (tableau 4). $\grave{A}$ Korhogo, les descripteurs intrasaisonniers : DSP, LSP, CPS, NJP, HMP, montrent les plus fortes variations au niveau des comparaisons des périodes. Le DSP de la période actuelle est retardé significativement de $26,1 \%$ (31 jours) par rapport aux périodes humide et sèche. La LSP de la période actuelle a subi un raccourcissement de $-31 \%$ (très significatif) par rapport à la période humide et un raccourcissement de -18\% (significatif) par rapport à la période sèche. Les CPS des périodes sèche et actuelle ont respectivement baissé de $-11 \%$ (significatif) et $26,9 \%$ (significatif) par rapport à la période humide. La période actuelle a montré une baisse significative de -
$17,8 \%$ par rapport à la période sèche. Les NJP des périodes sèche et actuelle sont respectivement en baisse de $-13,3 \%$ (significatif) et -15,7\% (significatif) par rapport à la période humide. Quant à la HMP de la période actuelle, elle a baissé significativement de $14,9 \%$ par rapport à la période humide et très significativement de $-16,7 \%$ par rapport à la période 1971-2000 (tableau 5). Les variations importantes à Ouangolodougou sont observées singulièrement au niveau de la comparaison faite entre période 19511970 et la période 1971-2000. Ainsi, la période sèche a montré un retard significatif du DSP de $-13,8 \%$ (18 jours) par rapport à la période humide. La LSP a montré une réduction significative de $-12,4 \%$. En revanche, le CPS et le NJP ont respectivement baissé de façon très significative de $-27,5 \%$ et de $-17,2 \%$ par rapport à la période humide (tableau 6). 
Dekoula et al., J. Appl. Biosci. 2018 Variabilité des descripteurs pluviométriques intrasaisonniers à impact agricole dans le bassin cotonnier de Côte d'Ivoire : zones de Boundiali, Korhogo et Ouangolo

Tableau 4II : Inter-comparaison entre les périodes de référence des descripteurs de la saison des pluies à Boundiali

\begin{tabular}{|c|c|c|c|c|}
\hline Variables & Comparaison & Moyennes & Variations (\%) & Probabilité ( $p)$ \\
\hline \multirow{3}{*}{ DSP } & Humide vs sèche & 10 mai vs 17 mai & 5,3 & 0.480 \\
\hline & Humide vs actuelle & 10 mai vs 21 avr. & $-14,5$ & 0,209 \\
\hline & Sèche vs actuelle & 17 mai vs 21 avr. & $-18,8$ & 0,095 \\
\hline \multirow{3}{*}{ FSP } & Humide vs sèche & 11 nov. vs 03 nov. & $-2,5$ & $0,048^{*}$ \\
\hline & Humide vs actuelle & 11 nov. vs 26 oct. & $-4,7$ & $0,046^{*}$ \\
\hline & Sèche vs actuelle & 03 nov. vs 26 oct. & $-2,3$ & 0,269 \\
\hline \multirow{3}{*}{ LSP } & Humide vs sèche & 189 vs 171 & $-9,5$ & 0,094 \\
\hline & Humide vs actuelle & 189 vs 190 & 0,5 & 0.967 \\
\hline & Sèche vs actuelle & 171 vs 190 & 11,1 & 0,298 \\
\hline \multirow{3}{*}{ CPS } & Humide vs sèche & 1401 vs 1190 & $-14,4$ & $0,046^{*}$ \\
\hline & Humide vs actuelle & 1401 vs 1060 & $-24,4$ & $0,040^{*}$ \\
\hline & Sèche vs actuelle & 1200 vs 1060 & $-11,6$ & 0,407 \\
\hline \multirow{3}{*}{ NJP } & Humide vs sèche & 71 vs 60 & $-15,5$ & $0,020^{*}$ \\
\hline & Humide vs actuelle & 71 vs 60 & $-15,5$ & 0,101 \\
\hline & Sèche vs actuelle & 60 vs 60 & 0,0 & 0,979 \\
\hline \multirow{3}{*}{ HMP } & Humide vs sèche & 20 vs 21 & $-5,0$ & 0,698 \\
\hline & Humide vs actuelle & 20 vs 18 & $-10,0$ & 0,293 \\
\hline & Sèche vs actuelle & 21 vs 18 & $-14,3$ & 0,344 \\
\hline \multirow{3}{*}{$\mathrm{ES}_{\mathrm{jn}}$} & Humide vs sèche & 7 vs 7 & 0,0 & 0,925 \\
\hline & Humide vs actuelle & 7 vs 10 & $-42,9$ & 0,161 \\
\hline & Sèche vs actuelle & 7 vs 10 & $-42,9$ & 0,145 \\
\hline \multirow{3}{*}{$E_{j t}$} & Humide vs sèche & 8 vs 8 & 0,0 & 0,982 \\
\hline & Humide vs actuelle & 8 vs 9 & 12,5 & 0,568 \\
\hline & Sèche vs actuelle & 8 vs 9 & 12,5 & 0,691 \\
\hline \multirow{3}{*}{$E_{\text {ju }}$} & Humide vs sèche & 5 vs 5 & 0,0 & 0,708 \\
\hline & Humide vs actuelle & 5 vs 5 & 0,0 & 0,600 \\
\hline & Sèche vs actuelle & 5 vs 5 & 0,0 & 0,911 \\
\hline \multirow{3}{*}{$\mathrm{ES}_{\mathrm{sp}}$} & Humide vs sèche & 4 vs 5 & 25,0 & 0,076 \\
\hline & Humide vs actuelle & 4 vs 5 & 25,0 & 0,244 \\
\hline & Sèche vs actuelle & 5 vs 5 & 0,0 & 0,911 \\
\hline
\end{tabular}

* tendance significative et ${ }^{* *}$ tendance très significative $(p<0,05)$ 
Dekoula et al., J. Appl. Biosci. 2018 Variabilité des descripteurs pluviométriques intrasaisonniers à impact agricole dans le bassin cotonnier de Côte d'Ivoire : zones de Boundiali, Korhogo et Ouangolo

Tableau 5 : Inter-comparaison entre les périodes de référence des descripteurs de la saison des pluies à Korhogo

\begin{tabular}{|c|c|c|c|c|}
\hline Variables & Comparaison & Moyennes & Variations (\%) & Probabilité $(p)$ \\
\hline \multirow{3}{*}{ DSP } & Humide vs sèche & 28 avr. vs 28 avr. & 0,0 & 0,940 \\
\hline & Humide vs actuelle & 28 avr. vs 29 mai & 26,1 & $0,037^{*}$ \\
\hline & Sèche vs actuelle & 28 avr. vs 29 mai & 26,1 & $0,025^{*}$ \\
\hline \multirow{3}{*}{ FSP } & Humide vs sèche & 09 nov. vs 03 nov. & $-1,6$ & 0,112 \\
\hline & Humide vs actuelle & 09 nov. vs 30 oct. & $-3,2$ & 0,145 \\
\hline & Sèche vs actuelle & 03 nov. vs 30 oct. & $-1,3$ & 0,537 \\
\hline \multirow{3}{*}{ LSP } & Humide vs sèche & 195 vs 189 & $-3,1$ & 0,389 \\
\hline & Humide vs actuelle & 195 vs 154 & $-21,0$ & $0,009^{* *}$ \\
\hline & Sèche vs actuelle & 189 vs 154 & $-18,0$ & $0,010^{*}$ \\
\hline \multirow{3}{*}{ CPS } & Humide vs sèche & 1216 vs 1082 & $-11,0$ & $0,031^{*}$ \\
\hline & Humide vs actuelle & 1216 vs 890 & $-26,9$ & $0,017^{*}$ \\
\hline & Sèche vs actuelle & 1083 vs 890 & $-17,8$ & $0,030^{*}$ \\
\hline \multirow{3}{*}{ NJP } & Humide vs sèche & 83 vs 72 & $-13,3$ & $0,030^{*}$ \\
\hline & Humide vs actuelle & 83 vs 70 & $-15,7$ & $0,042^{*}$ \\
\hline & Sèche vs actuelle & 72 vs 70 & $-2,8$ & 0,739 \\
\hline \multirow{3}{*}{ HMP } & Humide vs sèche & 14,8 vs 15 & 1,35 & 0,561 \\
\hline & Humide vs actuelle & 14,8 vs 12,6 & $-14,9$ & $0,048^{*}$ \\
\hline & Sèche vs actuelle & 15 vs 12,6 & $-16,7$ & $0,006^{* *}$ \\
\hline \multirow{3}{*}{$\mathrm{ES}_{\mathrm{jn}}$} & Humide vs sèche & 6 vs 6 & 0,0 & 0,777 \\
\hline & Humide vs actuelle & 6 vs 8 & 33,3 & 0,086 \\
\hline & Sèche vs actuelle & 6 vs 8 & 33,3 & 0,140 \\
\hline \multirow{3}{*}{$E_{\mathrm{jt}}$} & Humide vs sèche & 6 vs 7 & 16,7 & 0,276 \\
\hline & Humide vs actuelle & 6 vs 8 & 33,3 & 0,157 \\
\hline & Sèche vs actuelle & 7 vs 8 & 14,3 & 0,391 \\
\hline \multirow{3}{*}{$\mathrm{ES}_{\mathrm{au}}$} & Humide vs sèche & 5 vs 4 & $-20,0$ & 0711 \\
\hline & Humide vs actuelle & 5 vs 5 & 0,0 & 0,755 \\
\hline & Sèche vs actuelle & 4 vs 5 & 25,0 & 0,200 \\
\hline \multirow{3}{*}{$\mathrm{ES}_{\mathrm{sp}}$} & Humide vs sèche & 3 vs 4 & 33,3 & 0,051 \\
\hline & Humide vs actuelle & 3 vs 5 & 66,7 & 0,050 \\
\hline & Sèche vs actuelle & 4 vs 5 & 25,0 & 0,240 \\
\hline
\end{tabular}

* tendance significative et ** tendance très significative $(p<0,05)$ 
Dekoula et al., J. Appl. Biosci. 2018 Variabilité des descripteurs pluviométriques intrasaisonniers à impact agricole dans le bassin cotonnier de Côte d'Ivoire : zones de Boundiali, Korhogo et Ouangolo

Tableau 6 : Inter-comparaison entre les périodes de référence des descripteurs de la saison des pluies à Ouangolodougou

\begin{tabular}{|c|c|c|c|c|}
\hline Variables & Comparaison & Moyennes & Variations (\%) & Probabilité $(p)$ \\
\hline \multirow{3}{*}{ DSP } & Humide vs sèche & 9 mai vs 27 mai & 13,8 & $0,048^{*}$ \\
\hline & Humide vs actuelle & 9 mai vs 31 mai & 16,9 & 0,174 \\
\hline & Sèche vs actuelle & 27 mai vs 31 mai & 2,7 & 0,797 \\
\hline \multirow{3}{*}{ FSD } & Humide vs sèche & 03 nov. vs 28 oct. & $-1,6$ & 0,165 \\
\hline & Humide vs actuelle & 03 nov. vs 29 oct. & $-1,0$ & 0,573 \\
\hline & Sèche vs actuelle & 28 oct. vs 29 oct. & 0,3 & 0,768 \\
\hline \multirow{3}{*}{ LSP } & Humide vs sèche & 177 vs 155 & $-12,4$ & $0,041^{*}$ \\
\hline & Humide vs actuelle & 177 vs 152 & $-14,1$ & 0,147 \\
\hline & Sèche vs actuelle & 155 vs 152 & $-1,9$ & 0,888 \\
\hline \multirow{3}{*}{ CPS } & Humide vs sèche & 1143 vs 829 & $-27,5$ & $0,003^{* *}$ \\
\hline & Humide vs actuelle & 1143 vs 905 & $-20,8$ & 0,166 \\
\hline & Sèche vs actuelle & 829 vs 905 & 9,2 & 0,440 \\
\hline \multirow{3}{*}{ NJP } & Humide vs sèche & 58 vs 47 & $-17,2$ & $0,009^{* *}$ \\
\hline & Humide vs actuelle & 58 vs 53 & $-8,6$ & 0,441 \\
\hline & Sèche vs actuelle & 47 vs 53 & 12,8 & 0,372 \\
\hline \multirow{3}{*}{ HMP } & Humide vs sèche & 20,2 vs 18,2 & $-9,9$ & 0,181 \\
\hline & Humide vs actuelle & 20,2 vs 17,1 & $-15,3$ & 0,334 \\
\hline & Sèche vs actuelle & 18,2 vs 17,1 & $-6,0$ & 0,522 \\
\hline \multirow{3}{*}{$\mathrm{ES}_{\mathrm{jn}}$} & Humide vs sèche & 7 vs 8 & $-14,3$ & 0,385 \\
\hline & Humide vs actuelle & 7 vs 6 & $-14,3$ & 0,207 \\
\hline & Sèche vs actuelle & 8 vs 6 & $-25,0$ & 0,200 \\
\hline \multirow{3}{*}{$E_{\mathrm{jt}}$} & Humide vs sèche & 7 vs 9 & 28,6 & 0,117 \\
\hline & Humide vs actuelle & 7 vs 6 & $-14,3$ & 0,191 \\
\hline & Sèche vs actuelle & 9 vs 6 & $-33,3$ & 0,169 \\
\hline \multirow{3}{*}{$\mathrm{ES}_{\mathrm{au}}$} & Humide vs sèche & 5 vs 7 & $-40,0$ & 0,090 \\
\hline & Humide vs actuelle & 5 vs 5 & 0,0 & 1,00 \\
\hline & Sèche vs actuelle & 7 vs 5 & $-28,6$ & 0,362 \\
\hline \multirow{3}{*}{$E_{s p}$} & Humide vs sèche & 5 vs 6 & 20,0 & 0,05 \\
\hline & Humide vs actuelle & 5 vs 4 & $-20,0$ & 0,458 \\
\hline & Sèche vs actuelle & 6 vs 4 & $-33,3$ & 0,119 \\
\hline
\end{tabular}

* tendance significative et ${ }^{* *}$ tendance très significative $(p<0,05)$

\section{DISCUSSION}

La saison des pluies est depuis quelques années confrontée à des anomalies issues des perturbations des descripteurs intrasaisonniers qui caractérisent sa qualité. En effet, l'analyse des DIS sur la période 19512000 a révélé des variabilités spatio-temporelles dans la zone de production cotonnière. Le début de la saison des pluies oscille entre le 1er mai à Korhogo (Centre de la zone) et le 22 mai à Ouangolodougou (Nord-Est de la zone). Cette évolution traduit un lien étroit entre la dynamique de la mousson qui pénètre initialement par le Sud-Ouest dans le secteur de Tabou (Kanga, 2017). Le démarrage progressif de la saison des pluies s'explique donc par le mouvement en zone soudanienne de la mousson humide du Sud-Ouest vers le Nord-Est. Ces résultats corroborent ceux de Noufé et al. (2015) qui ont noté une évolution latitudinale du démarrage de la saison des pluies utiles dans l'Est ivoirien. Adewi et al. (2010) ont abouti aux mêmes résultats dans leurs travaux sur l'évolution des saisons des pluies potentiellement utiles au Togo. La fin de la saison des pluies (FSP) survient en moyenne dans la première décade $\mathrm{du}$ mois de novembre avec une tendance de plus en plus précoce de près de 2 jours par décennie. Ces résultats complètent ceux de Noufé et al. (2015) et Dekoula et al. (2018) qui ont observé des fins de saison de plus en plus précoces 
respectivement à Bouna au Nord-Est de la Côte d'Ivoire (7 jours) et dans la zone cotonnière ivoirienne (entre 2 et 12 jours). La longueur de la saison des pluies (LSP) comprise entre 162 jours et 188 jours, traduit une tendance significative au raccourcissement de près de 6 jours par décennie à Ouangolodougou. Ce raccourcissement de la LSP a été également mentionné dans les travaux de plusieurs auteurs en Côte d'Ivoire (Brou, 2005; Goula et al., 2010). Ces résultats traduisent les perturbations que subissent les calendriers culturaux indiquées par Diomandé et al. (2013) dans la zone de contact savane-forêt. L'évolution spatiale du cumul pluviométrique saisonnier (CPS) et du nombre de jours de pluie (NJP) suit un gradient croissant est-ouest. En effet, les valeurs passent de faible à forte de Ouangolodougou (NordEst) à Boundiali (Ouest). Aussi, les tendances à la baisse sont-elles très significatives au Nord-Est et non significatives à l'Ouest de la zone d'étude. Ce résultat est similaire à ceux de Soro et al. (2013) dans le bassin-versant du Haut Bandama au Nord de la Côte d'Ivoire. L'évolution de la hauteur moyenne des précipitations journalières (HMP) ne traduit pas le même gradient que celui du CPS et du NJP. Cependant, la baisse est tout de même significative à Boundiali. Les différentes tendances observées témoignent d'une baisse générale de la pluviométrie dans la zone cotonnière étudiée avec des particularités locales au niveau de certains DIS. Ces résultats sont similaires à ceux obtenus par Kouassi et al. (2010) dans le bassin-versant du N'zi (Bandama) en Côte d'Ivoire et par Noufé et al. (2015) dans l'Est ivoirien. Aussi Bambara et al. (2016) ont-ils abouti aux mêmes résultats au Burkina Faso. Les séquences sèches (SS) au cours des mois de développement des cultures, ne dépassent pas 8 jours par mois. Cependant, l'occurrence des SS en fin de mois et en début du mois suivant, pourrait être dommageable pour les cultures si elles coincident avec la phase de floraison. En effet, pour les cultures comme le coton un jour sec pendant la période de floraison engendre une perte de rendement de $16 \mathrm{~kg} / \mathrm{ha} / \mathrm{jour}$ (Milroy et al., 2002). Le test de Pettitt (1979) a permis de mettre en évidence, la non-stationnarité au niveau des DIS. Les ruptures les plus significatives détectées par ce test sont situées entre 1968 et 1979. Ce résultat s'inscrit globalement dans la période de rupture pluviométrique observée dans la majorité des pays de l'Afrique de l'Ouest (Paturel et al., 1997, Servat et al., 1999; Ozer et al., 2009) et en Côte d'Ivoire en particulier (Brou, 2005; Soro et al., 2011). Toutefois, selon Noufé (2011) si la majorité des auteurs s'accorde à reconnaître que la rupture s'est globalement produite en Côte d'Ivoire autour de 1970, ce n'est qu'à titre indicatif. Pour preuve, les dates de décrochage trouvées ici, sont antérieures (1968) ou postérieures (1979) à cette date. En outre, dans les localités d'étude, les descripteurs tels que la LSP, le CPS et le NJP ont des dates de rupture qui coïncident ou différent de 2 années. Ces résultats traduisent le lien étroit qui existe entre ces trois descripteurs. Dès lors, la tendance pluviométrique à la baisse observée à partir des années 1970 peut être directement associée à une modification des DIS conditionnant la qualité de la saison agricole. Boyard et al. (2013) ont pu mettre en exergue une forte relation entre les rendements potentiels du sorgho et les descripteurs LSP et CPS. Par conséquent, les variations des DIS constituent une contrainte pour ; la planification des travaux champêtres, le calage des cycles culturaux et le rendement des cultures en agriculture pluviale. L'analyse comparative des périodes montre une variation des descripteurs de la saison des pluies dans les différentes localités. À Boundiali, la FSP, le CPS et le NJP montrent les variations les plus importantes entre les périodes; humide et sèche, et humide et actuelle. Ce résultat traduit une dégradation au niveau de ces descripteurs après la rupture et qui perdure actuellement. À Korhogo, la comparaison des trois périodes montre des variations plus importantes au niveau des DSP, LSP, CPS, NJP et HMP. Les niveaux très significatifs traduisent une baisse pluviométrique après la période humide qui s'est accrue pendant la période actuelle. En revanche à Ouangolodougou, seule la comparaison période humide et période sèche a montré des variations importantes au niveau des descripteurs DSP, LSP et CPS. Cependant les niveaux de significativité $>5 \%$ pour la comparaison entre la période sèche et la période actuelle traduisent des conditions presqu'identiques entre ces deux périodes. Plusieurs acteurs ont abouti à des résultats similaires. C'est le cas de Goula et al., (2010) qui ont montré une réduction de la durée de la saison des pluies de 10 à 20 jours dans plusieurs régions de la Côte d'lvoire après 1970. Noufé et al. (2015) indiquent des débuts de saison des pluies de plus en plus tardifs et des fins de saison pluvieuse de plus en plus précoces dans l'Est du pays après la rupture de 1970. Kouassi et al. (2010) font remarquer la baisse de la pluviométrie et des jours de pluie dans le bassin-versant du N'zi-Bandama. Les travaux d'Adewi et al., (2010) et de Bambara et al., (2016) respectivement au Togo et au Burkina Faso ont 
abouti au même constat. Toutes ces situations traduisent une incertitude grandissante quant à l'utilité des DIS pour la production agricole (Alhassane et al., 2013). En effet, la baisse importante et continue des

\section{CONCLUSION}

L'objectif de cette étude était de caractériser l'évolution des descripteurs pluviométriques intrasaisonniers dans la zone de production cotonnière, à travers l'analyse des tendances, des stationnarités et l'inter-comparaison des périodes humide (1951-1970), sèche (1971-2000) et actuelle (2011-2016). Les résultats ont permis de mettre en évidence une perturbation des DIS entre 1970 et 2000. Cette perturbation observée s'est prolongée voire accentuée entre 2011-2016. Ainsi, la variation de la pluviométrie observée ces dernières décennies a provoqué des perturbations des descripteurs intrasaisonniers garants de la qualité de la saison agricole. Les tendances décennales montrent un retard dans l'installation des pluies, une fin précoce et un raccourcissement de la saison des pluies. Le cumul saisonnier, le nombre de jours pluvieux et la hauteur moyenne des précipitations journalières indiquent une tendance à la baisse. Quant aux séquences sèches

\section{RÉFÉRENCES BIBLIOGRAPHIQUES}

Addinsoft. 2016. XLSTAT 2016: Data Analysis and Statistical Solution for Microsoft Excel. Paris, France.

Adewi E, Badameli K, Dubreuil V, 2010. Évolution des saisons des pluies potentiellement utiles au Togo de 1950 à 2000. Climatologie $7:$ 89-107.

Aguiar L. A. A., 2009. Impact de la variabilité climatique récente sur les écosystèmes des niayes $\mathrm{du}$ Sénégal entre 1950 et 2004. Thèse; Université du Québec à Montréal, Québec, 185p.

Ahoussi K. E., Koffi Y. B., Kouassi AM., Soro G., Soro N. et Biémi J. 2013. Étude de la variabilité hydroclimatique et de ses conséquences sur les ressources en eau du Sud forestier et agricole de la Côte d'Ivoire : cas de la région d'Abidjan-Agboville. International Journal of Pure \& Applied Bioscience. 1(6) : 30-50.

Alhassane A., Salack S., Ly M., Lona I., Traoré, S. B., et Sarr B., 2013. Évolution des risques agroclimatiques associés aux tendances récentes du régime pluviométrique en Afrique de l'Ouest soudano-sahélienne. Science et changements planétaires/Sécheresse, 24(4) : 282-293. DOI : $10.1684 / \mathrm{sec} .2013 .0400$
CPS et du NJP observée dans l'ensemble du pays a des conséquences sur la qualité de la saison des pluies utiles à l'agriculture.

des mois de juin à septembre, elles varient peu. Par ailleurs, les résultats indiquent des ruptures au niveau des descripteurs intrasaisonniers comprises entre 1968-1979. La comparaison des périodes a, quant à elle, montré une dégradation des conditions climatiques au cours de la période sèche. Cette détérioration de la majorité des descripteurs a perduré et s'est même accentuée dans la période actuelle. Les séquences sèches ont certes peu évolué mais pourraient être un facteur limitant si elles apparaissent en fin de mois et en début du mois suivant. Dans ce contexte de variabilité observée au niveau des descripteurs intrasaisonniers, la planification agricole est de plus en plus difficile. Ainsi, la caractérisation de la saison des pluies s'avère être une étape nécessaire dans la réalisation des différentes stratégies adaptatives face aux perturbations climatiques en vue d'améliorer les rendements agricoles.

Balliet R., Saley M. B., Eba E. L. A., Sorokoby V. M., N'Guessan Bi H. V., N'Dri A. O., Djè B. K. et Biémi J., 2016. Évolution Des Extrêmes Pluviométriques Dans La Région Du Gôh (Centre-Ouest De La Côte d'Ivoire) European Scientific Journal. Edition 12 (23) : 1857-7881. DOI: 10.19044/esj.2016.

Bambara, D., Thiombiano, A., \& Hien, V., 2016. Changements climatiques en zones nordsoudanienne et sub-sahélienne du Burkina Faso: comparaison entre savoirs paysans et connaissances scientifiques. Revue d'écologie, 71 (1) : 35-58

Boyard-Micheau J., 2013. Prévisibilité potentielle des variables climatiques à impact agricole en Afrique de l'Est et application au sorgho dans la région du mont Kenya. Thèse de doctorat, Université de Bourgogne, France, 338p.

Boyard-Micheau J., Camberlin P. et Philippon N., 2013. Sensibilité des rendements agricoles potentiels aux paramètres climatiques et environnementaux dans la région du mont Kenya en Afrique de l'Est. M. Boko, E.W. Vissin \& F. Afouda. 26e colloque de I'Association Internationale de Climatologie, 
Sep, Cotonou, Bénin. Association Internationale de Climatologie, Climat, agriculture et ressources en eau d'hier à demain, pp.122-128.

Brou T. Y., 1997. Analyse et dynamique de la pluviométrie en milieu forestier ivoirien: recherche de corrélations entre les variables climatiques et les variables liées aux activités anthropiques. Doctorat $3^{e}$ cycle, Université de Cocody-Abidjan, $210 \mathrm{p}$.

Brou T. Y., 2005. Climat, mutations socio-économiques et paysages en Côte d'Ivoire. Mémoire de synthèse des activités scientifiques présenté en vue de l'obtention de l'Habilitation à Diriger des Recherches, Université des Sciences et Techniques de Lille, France, $212 \mathrm{p}$.

Dekoula C. S., Kouame B., N'goran E. K., Yao, F. G., Ehounou J. N., et Soro, N., 2018. Impact de la variabilité pluviométrique sur la saison culturale dans la zone de production cotonnière en Côte d'Ivoire. European Scientific Journal, ESJ, 14(12) : 143-156

Diomandé M., Dongo K., Djé K. B., Kouadio K. K. H., Koné D., Biémi, J., et Bonfoh, B., 2013. Vers un changement du calendrier cultural dans l'écotone forêt-savane de la Côte d'Ivoire. Agronomie Africaine, 25(2), 133-147.

Goula B. T., Srohourou B., Brida, A., N'zué K. A. et Goroza G. 2010. Determination and variability of growing seasons in Côte d'lvoire. Int J Eng Sci, 2(11) : 5993-6003.

Intercoton 2018. http://www.intercoton.org/statistiques. php?article $=$ Statistiques\&mark $=5$

Kanga K. H. M., 2017. Sècheresse et vulnérabilité socio-spatiale dans le quart nord-est de la Côte d'lvoire. Thèse unique de doctorat, Université Alassane Ouattara, Bouaké, 347p.

Kendall M. G., 1975. Rank Correlation Methods. 4th Edition, Charles Griffin, London, $202 p$

Koné B., 2007. Utilisation des données pour l'étude de la fertilité potentielle des sols ferralitiques audessus de la latitude $7^{\circ} \mathrm{N}$ de la Côte d'Ivoire. Thèse unique, Université de Cocody, 146p.

Kouassi A. M., Kouame K. F., Koffi Y. B., Dje K. B., Paturel J. E. et Oulare S., 2010. Analyse de la variabilité climatique et de ses influences sur les régimes pluviométriques saisonniers en Afrique de l'Ouest : cas du bassin versant du N'zi (Bandama) en Côte d'Ivoire, Cybergeo : European Journal of Geography, Environnement, Nature, Paysage, document
513, mis en ligne le 07 décembre 2010, consulté le 16 décembre 2013. URL: http://cybergeo.revues.org/23388.

Le Guen T., 2004. Le développement agricole et pastoral du Nord de la Côte-d'Ivoire : problèmes de coexistence. Les Cahiers d'Outre-Mer. Revue de géographie de Bordeaux, 57(226-227), 259-288.

Mann H. B., 1945. Nonparametric Tests against Trend. Econometrica, 13 (3): 245-259. DOI: 10.2307/1907187

Milroy S., Goyne P. et Larsen D., 2002. Irrigation Scheduling of Cotton. Cotton Information Sheet. Australian Cotton co-operative Research Centre.

Moron V., Robertson A. W., Ward M. N. and Camberlin P., 2007. Spatial coherence of tropical rainfall at the regional scale. Journal of Climate, 20(21), 524a4-5263. DOI: https://doi.org/10.1175/2007JCLI1623.1

Noufé D., Lidon B., Mahé G., Servat E. et Chaléard J. L. (2015). Impact de l'évolution des conditions agroclimatiques sur les systèmes de culture à base de banane plantain : le cas de l'Est ivoirien. VertigO la revue électronique en sciences de l'environnement, 15 (1) : 1-15.

Noufé D., Lidon B., Mahé G., Servat E., Brou Y. T., Bi Zueli K., et Chaléard J. L., 2011.Variabilité climatique et production de maïs en culture pluviale dans l'est Ivoirien. Hydrological sciences journal, 56(1), 152-167. DOI: https://doi.org/10.1080/02626667.2010.545247

O.M.M., 1990. Guide des pratiques climatologiques, (100). Genève, Suisse, $515 p$.

Ozer P., Hountondji Y.C., Gassani J., Djaby B., et de Longueville $F .$, 2014. Evolution récente des extrêmes pluviométriques en Mauritanie (1933-2010). In: Camberlin P., Richard Y. (Eds.), Actes du 27e Colloque International de I'Association Internationale de Climatologie. Dijon, France: Université de Bourgogne, pp.394 -400.

Paturel J. E., Servat E., Kouame B., Lubes H., Masson J. M., Boyer J. F., Travaglio M. et Marieu B., 1997. Variabilité pluviométrique en Afrique humide le long du Golfe de Guinée. Approche régionale intégrée. Documents Techniques en Hydrologie, UNESCO, Paris, 16 : 1-31.

Pettitt A. N., 1979. A non-parametric approach to the change-point problem. Applied statistics, 28 : 126-135. DOI : $10.2307 / 2346729$ 
Sarr B., 2007. Manuel d'utilisation destiné aux ingénieurs en agrométéorologie. Centre Régional AGHYMET, Niamey, 72p.

Servat E., Paturel J. E., Lubes-Niel H., Kouamé B., Masson J. M., Travaglio M. et Marieu B., 1999. De différents aspects de la variabilité de la pluviométrie en Afrique de l'ouest et centrale non sahélienne. Revue des sciences de l'eau, 12 (2): 363-387.

Sivakumar M., 1988. Predicting rainy season potential from the onset of rains in the southern sahelian and soudanian climatic zone of West Africa. Agricultural and forest meteorology, 42: 295-305.

Soro T. D., Kouakou D. B., Kouassi A. E., Soro G., Kouassi M. A., Kouadio E. K., Oga Y. M. S. et Soro, N., 2013. Hydroclimatologie et dynamique de l'occupation du sol du bassin versant du Haut Bandama à Tortiya (Nord de la Côte d'lvoire). [VertigO] La revue électronique en sciences de l'environnement, 13(3).

Soro T. D., Soro N., Oga Y. M. S., Lasm T., Soro G., Ahoussi K. E. et Biémi J., 2011. La variabilité climatique et son impact sur les ressources en eau dans le degré carré de Grand-Lahou (Sud-Ouest de la Côte d'Ivoire). Physio-Géo Géographie Physique et Environnement, Vol.5, pp.55-73. DOI: 10.4000/physiogeo.1581

Stern R., Rijks D., Dale I., Knock J., 2006. INSTAT+ for Windows V3.036 Statistical Services Center, University of Reading: Reading.

Sultan B., Baron C., Dingkuhn M., Sarr B. et Janicot S., 2005 : La variabilité climatique en Afrique de l'Ouest aux échelles saisonnière et intrasaisonnière. II : applications à la sensibilité des rendements agricoles au Sahel. Sécheresse, 16(1), pp. 23-33. 